\title{
La enseñanza de la Psiquiatría en México
}

\author{
Juan Ramón de la Fuente, Gerhard Heinze Martin
}

\section{SUMMARY}

Our country was the pioneer in the care of people with mental illnesses in America. San Hipólito's Hospital and the general asylum La Castañeda attest to this. Although the knowledge and therapeutic resources were initially limited, advances in psychiatric nosology gradually emerged. A number of prominent Mexican physicians found their way into the field of mental health in the early 1940s.

Psychoanalysis left a deep mark on Western culture. However, the discovery of psychotropic drugs and the establishment of a modern psychiatric vision changed the teaching of psychiatry, emphasizing its importance in the medical career and the specialty. On the other hand, in recent decades the development of neuroscience and the social sciences has brought about progress in the classification of mental illnesses and their treatment.

Today, resources for mental health care in our country are low and are particularly concentrated in the third level of medical care. Associated with this, we have about four thousand psychiatrists and child and adolescent psychiatrists, who turn out to be insufficient for they are concentrated in large cities and the demand is growing. There exists a great interest in integrating mental health into the primary care network health. In our country, we have twenty offices with structured psychiatric specialization programs in sixteen universities.

The future of psychiatry looks promising. The ability to relate more closely mental disorders with biology by imaging techniques, genomics and proteomics opens new horizons for therapeutic, keeping current psychological and social dimensions for clinical attention.

Key words: Psychiatry, specialty, teaching.
RESUMEN

Nuestro país fue pionero en la atención de las personas con enfermedades mentales en América; el Hospital de San Hipólito y el manicomio general de "La Castañeda" dan cuenta de ello. Aunque en un inicio los conocimientos y la terapéutica fueron limitados, gradualmente surgieron avances en la nosología psiquiátrica. Una serie de prominentes médicos mexicanos se abrió paso en el campo de la salud mental a principios de la década de 1940.

El psicoanálisis dejó una huella profunda en la cultura occidental, sin embargo, el descubrimiento de los medicamentos psicotrópicos y el establecimiento de la psiquiatría moderna cambiaron la visión en la enseñanza, remarcando su importancia en la carrera de medicina y en la especialidad. Por otro lado, el desarrollo de las neurociencias y de las ciencias sociales en las últimas décadas ha traído consigo un avance en la clasificación de las enfermedades mentales y su tratamiento.

Hoy en día los recursos destinados a la atención de la salud mental en nuestro país son bajos y se concentran especialmente en el tercer nivel de atención. Asociado a ello contamos con cerca de cuatro mil psiquiatras y paidopsiquiatras, los cuales son insuficientes ya que se concentran en las grandes ciudades y la demanda es cada vez mayor por lo que hay gran interés en integrar a la salud mental a las redes de atención primaria de la salud. En nuestro país tenemos veintidós sedes de especialización psiquiátrica con programas estructurados que se imparten en dieciséis universidades.

El futuro de la psiquiatría es prometedor, la posibilidad de relacionar con mayor precisión a los trastornos mentales con la biología gracias a las técnicas de imagen, la genómica y la proteómica abren nuevos horizontes para la terapéutica, manteniendo vigentes las dimensiones psicológicas y sociales para la atención clínica.

Palabras clave: Psiquiatría, especialidad, enseñanza.

\section{ANTECEDENTES HISTÓRICOS}

México se adelantó a todos los demás países del continente americano en la atención hospitalaria de los enfermos mentales. En 1566 Fray Bernardino Álvarez fundó el hospital de San Hipólito, primero en su género dedicado al cuidado de tales enfermos en las Américas. No obstante, la obra de este ilustre precursor realmente no incide en el panorama general. Los enfermos mentales eran con frecuencia aban- donados por sus familias, deambulaban por las calles mal alimentados y, en el mejor de los casos, eran guardados en espacios improvisados al amparo de algún personaje o agrupación compasiva.

En 1910, unos meses antes de que estallara la Revolución mexicana, el presidente Porfirio Díaz inauguró en los terrenos de la hacienda La Castañeda, en el sur de la Ciudad de Méxi$\mathrm{co}$, el manicomio general. Justo es decir que su edificio estuvo a la altura de las preferencias arquitectónicas de la época, y lo

Departamento de Psiquiatría y Salud Mental, Facultad de Medicina, Universidad Nacional Autónoma de México.

Correspondencia: Dr. Gerhard Heinze M. Edificio "F", Circuito Exterior y Cerro del Agua s/n. Facultad de Medicina, Ciudad Universitaria, Coyoacán, 04510 , México, DF. Email: gheinze@prodigy.net.mx; heinzeg@liceaga.facmed.com.mx

Recibido primera versión: 29 de agosto de 2014. Segunda versión: 26 de septiembre de 2014. Aceptado: 2 de octubre de 2014 
mismo puede decirse de los criterios médicos y administrativos que lo rigieron. La Castañeda fue la cuna de la psiquiatría pública mexicana, y, aún cuando se carecía de conocimientos y de recursos terapéuticos efectivos, la atención que ahí se prestaba no era necesariamente inferior a la que se ofrecía en otros países. Simplemente no había otras opciones. ${ }^{1}$

Los limitados conocimientos y recursos terapéuticos efectivos hasta bien entrado el siglo $X X$, contribuyeron al desarrollo de temores y prejuicios de la sociedad en general hacia las enfermedades mentales. Lo lamentable es que en algunos sectores tales prejuicios aún subsistan.

Si bien en 1783, en un acto lleno de simbolismo, Philippe Pinel liberó de las cadenas a los pacientes del asilo de Bicêtre y las pacientes internadas en el hospital de La Salpêtrière, en París, y estableció ahí el "tratamiento moral", a pesar de que había sido advertido por el movimiento revolucionario francés que era peligroso esconder entre ellos a los "enemigos de la Revolución", el gesto no tuvo mayor trascendencia, porque durante el siglo XIX la atención de los enfermos psiquiátricos consistía en baños de agua fría y sujeción con camisas de fuerza a los excitados, vigilando el deterioro progresivo de los afectados.

Ciertamente hubo algunos avances en la nosología psiquiátrica, sobre todo en la diferenciación de la esquizofrenia y la enfermedad maniaco-depresiva, en el estudio de la histeria, de los trastornos depresivos y de la ansiedad, pero la terapéutica seguía siendo muy limitada.

A principio de la década de los años 1930, ocurrieron desarrollos que ampliaron significativamente el tratamiento de algunos trastornos mentales severos. Por un lado, Sakel, en Viena, introdujo el uso de la insulina para producir estados comatosos en el tratamiento de las psicosis esquizofrénicas; y por el otro, Von Meduna, en Budapest, desarrolló la inducción de crisis convulsivas con metrazol con propósitos similares. Un poco después, Cerletti y Bini, en Roma, desarrollaron el electrochoque y Moniz, en Lisboa, realizó la primera lobotomía prefrontal, lo cual abrió el camino a la psicocirugía.

Mientras tanto, en nuestro país, como consecuencia de la Guerra civil española, y como parte de ese injerto formidable de talento que nos llegó, personificado en miles de trasterrados, algunos distinguidos neurólogos y psiquiatras encontraron en México terreno fértil para continuar su desarrollo profesional.

Gonzalo Lafora, Wenceslao López Alvo y Federico del Roncal, cada uno de ellos ya con prestigio, perfeccionaron en nuestro medio sus habilidades clínicas. Un eminente neuropatólogo español que llegó a México en 1940, fue Dionisio Nieto, quien desarrolló buena parte de sus trabajos en el hoy Instituto de Investigaciones Biomédicas de la Universidad Nacional Autónoma de México. Sobresalió en el campo de la psiquiatría biológica y también trabajó en el área clínica en el manicomio de La Castañeda. Algunos de sus principales alumnos y colaboradores fueron Agustín Caso y Augusto Fernández-Guardiola.
Entre 1933 y 1952, el escenario académico de la psiquiatría en México estuvo representado por un selecto grupo de médicos que sobresalieron por sus aportaciones y dedicación a los enfermos con trastornos mentales y neurológicos. Entre los que destacaron: Manuel Guevara Oropeza, Samuel Ramírez Moreno, Leopoldo Salazar Viniegra, Guillermo Dávila, Martín Ramos Contreras, Raúl González Enríquez, Edmundo Buentello, Alfonso Millán y Mario Fuentes Delgado. Fueron ellos quienes realmente desarrollaron la asistencia médica de los enfermos mentales tanto a nivel privado como en instituciones públicas. En 1946, por ejemplo, González Enríquez fundó la primera Unidad de Psiquiatría del Instituto Mexicano del Seguro Social. Además, sembraron vocaciones y cultivaron la enseñanza.

Sobresalió en la docencia Samuel Ramírez Moreno, quien fuera también el primer Director de Salud Mental en la Secretaría de Salubridad y Asistencia y quien siempre estuvo al tanto de los avances de la psiquiatría tanto de Europa como de los Estados Unidos. Fundó un hospital psiquiátrico privado con adiestramiento clínico para los médicos interesados en la especialidad y estimuló a sus discípulos a completar sus estudios en el extranjero. Fue Secretario General de la UNAM. ${ }^{2}$

En 1952, siendo Raoul Fournier Villada Director de la Facultad de Medicina de la Universidad Nacional Autónoma de México y un promotor de la medicina psicológica, se separaron los programas de enseñanza de la neurología y la psiquiatría, convirtiéndose cada una en disciplinas independientes. Fue en esta época que se estableció el primer curso formal de adiestramiento en Psiquiatría, con reconocimiento de la Escuela de Graduados de la UNAM.

En 1955, a iniciativa de Alfonso Millán se creó, en la propia Facultad, el Departamento de Psicología Médica, Psiquiatría y Salud Mental, como parte de una reforma tendiente a orientar la enseñanza de la medicina con un sentido psicológico y humanístico. En el plan de estudios se incluyeron las materias de psicología médica y medicina humanística, que posteriormente cambiaron a psicología médica I y II, y que actualmente, se denominan, respectivamente, introducción a la salud mental y medicina psicológica. ${ }^{3}$

En esa misma década, se desarrollaron los primeros tratamientos farmacológicos con fenotiazinas e inhibidores de la monoaminoxidasa, mucho más eficaces para las principales patologías mentales: la esquizofrenia, la depresión, los trastornos de ansiedad, entre otros. La psicofarmacología cambió radicalmente el pronóstico de muchos enfermos psiquiátricos y mejoraron las posibilidades de reintegrarlos nuevamente a la sociedad y a sus familias.

Entre 1960 y 1970, la Secretaría de Salubridad y Asistencia estableció una red de 11 hospitales, nueve de ellos hospitales-granjas, en sustitución del ya obsoleto y sobrepoblado manicomio general. Las nuevas instalaciones representaron un avance que, sin embargo, no logró consolidarse por falta de recursos técnicos y económicos. No obstante, destacan el 
hospital Fray Bernardino Álvarez y el hospital Psiquiátrico Infantil Juan N. Navarro, que tuvieron y mantienen una función importante tanto para la enseñanza como para fines asistenciales.

En los años subsecuentes, adquirió mayor relevancia la corriente que puso énfasis en el estudio de los enfermos mentales en su contexto social. Es decir, la atención a los enfermos incorporó a la familia, al grupo social, a la comunidad. Entre quienes hicieron aportaciones significativas desde esta perspectiva conviene recordar a Guillermo Calderón Narváez, Héctor Tovar y Carlos Pucheu, entre otros.

En 1970, Ramón de la Fuente Muñiz, considerado el fundador de la psiquiatría moderna en México, creó el primer servicio abierto de psiquiatría en un hospital general (el Hospital Español de México). Éste sigue vigente y se mantiene como uno de los principales centros de adiestramiento en psiquiatría dentro del contexto de la medicina hospitalaria. Como Profesor Titular del curso de posgrado en psiquiatría de la Facultad de Medicina de la UNAM, el maestro De la Fuente formó a un gran número de psiquiatras que hoy ejercen su especialidad en toda la Republica Mexicana y en otros países de Centro y Sudamérica. Fue el presidente del Congreso Mundial de Psiquiatría que se celebró en México en 1971, y creó, en 1972, el Centro Universitario de Salud Mental, sede del Departamento de Psiquiatría y Salud Mental de la Facultad de Medicina de la UNAM. Antes había fundado, en 1966, la Asociación Psiquiátrica Mexicana, y unos años después, el Consejo Mexicano de Psiquiatría. Profesor Emérito y Doctor Honoris Causa por la Universidad, fue el autor de libros clásicos entre los que sobresale Psicología Médica, reeditado y reimpreso decenas de veces por el Fondo de Cultura Económica. Dicha obra ha sido un texto irremplazable durante décadas en las escuelas de medicina de México y otros países, y contribuyó al reconocimiento cabal de la psiquiatría como rama de la medicina en nuestro país. ${ }^{4}$

En 1979 fundó el Instituto Mexicano de Psiquiatría, ahora Instituto Nacional de Psiquiatría que lleva su nombre, y creó la revista Salud Mental, órgano oficial del Instituto, acreditada y reconocida internacionalmente, después de 37 años de publicarse en forma ininterrumpida.

\section{PSIQUIATRÍA Y MEDICINA}

La enseñanza de la psiquiatría como parte de la enseñanza de la medicina en México se inició hacia finales del siglo XIX. El doctor Miguel Alvarado, Director del hospital para enfermos mentales de La Canoa daba algunas clases a sus alumnos al lado del enfermo e impartía, dentro del curso de "perfeccionamiento", en la Escuela de Medicina, algunas lecciones sobre enfermedades mentales.

A principios del siglo pasado Juan Peón del Valle y, un poco después, Enrique O. Aragón, también impartieron clases sobre el tema, pero fue en realidad, hasta 1922, cuando el doctor José Meza Gutiérrez impartió un curso de psiquiatría en el $6^{\circ}$ año de la carrera de medicina como materia optativa. ${ }^{5}$

Las primeras residencias hospitalarias en psiquiatría se iniciaron en el manicomio de La Castañeda en 1948. Había también cursos formales en los sanatorios privados de los doctores Rafael Lavista y Samuel Ramírez Moreno. Más adelante, en la década de los años 1950 se desarrollaron cursos en el Hospital Central Militar, se fortalecieron los de la Facultad de Medicina de la UNAM y se iniciaron en la Universidad Autónoma de Nuevo León.

En 1951, la UNAM estableció un curso formal de psiquiatría a iniciativa de Raúl González Henríquez y Guillermo Dávila con una duración de dos años. Este curso se ha venido perfeccionando a lo largo del tiempo y de manera coordinada se imparte en diversos centros hospitalarios tanto de la Secretaría de Salud como de las instituciones de seguridad social y del sector privado. ${ }^{6}$

En los años subsecuentes empezó a haber desarrollos importantes en la enseñanza de la psiquiatría en diversas entidades federativas. Se deben reconocer los esfuerzos realizados por Manuel Camelo Camacho y Rubén Tamez Garza en Monterrey; de Wenceslao Orozco, Fernando de la Cueva y Mario Saucedo en Guadalajara; de Ángel Ortiz Escudero en León; de Ignacio Rivero Blumenkron en Puebla así como de Antonio de la Maza y Everardo Neumann en San Luis Potosí, por mencionar algunos de los pioneros de la educación psiquiátrica universitaria en nuestro país. ${ }^{5}$

Los retos en la enseñanza de la psiquiatría, al igual que ocurre en otras especialidades de la medicina, consisten en poder incorporar oportunamente los avances científicos que nutren y modifican el campo propio de cada disciplina. En el caso de la psiquiatría, los avances han sido importantes tanto en los aspectos biológicos como en los psicológicos y sociales.

En los años más recientes, las neurociencias han tenido un desarrollo formidable y han modificado substancialmente la forma como entendemos y tratamos muchos de los trastornos mentales. A los fármacos psicotrópicos iniciales, que ya mostraban una inobjetable eficacia terapéutica, se han agregado otras moléculas capaces de controlar las alteraciones del estado de ánimo y de la percepción de la realidad de una manera mucho más selectiva. Esto ha permitido, entre otros beneficios, la reinserción social de muchos enfermos que antes debían estar confinados a centros hospitalarios. ${ }^{6}$

Pero junto con los avances de la biología, las ciencias sociales también han aportado conocimientos fundamentales que inciden de manera directa o indirecta en la prevención, el diagnóstico y el tratamiento de muchos enfermos mentales. El concepto mismo de etiopatogenia en psiquiatría, al igual que ocurre en otras especialidades médicas, ha cambiado, y ha adquirido una connotación multifactorial que propicia el desarrollo de modelos integrales más acordes con la realidad. De manera análoga, las comunidades terapéuticas y otros modelos de rehabilitación psicosocial, juegan un papel importante en el complejo proceso por el que 
muchos enfermos deben transitar para alcanzar su plena y productiva reinserción en el ámbito familiar y social.

Si bien el psicoanálisis ha dejado de tener la influencia que en una época tuvo en el contexto de la psiquiatría, conviene recordar que en nuestro país, a principios de la segunda mitad del siglo pasado, regresaron a México algunos jóvenes psicoanalistas formados en la tradición freudiana, entre los que destacaron Santiago Ramírez y Ramón Parres. En la misma época, invitado por la UNAM, llegó a México Erich Fromm, precedido de fama internacional por sus aportaciones a la corriente humanista y social en el campo del psicoanálisis. Hoy en día el psicoanálisis es una disciplina autónoma, un tanto apartada de la medicina pero que, sin duda, ha dejado una huella profunda en la cultura occidental.

Los esfuerzos realizados por varias generaciones de psiquiatras distinguidos e interesados en la enseñanza de la psiquiatría, alcanzaron un merecido reconocimiento en 1972, con la Fundación del Consejo Mexicano de Psiquiatría reconocido por la Academia Nacional de Medicina. Al igual que otros Consejos, su función es certificar a quienes han tenido un adiestramiento adecuado y son aptos para el ejercicio profesional de la especialidad. ${ }^{5}$

\section{LA SALUD MENTAL EN MÉXICO}

En el estudio de la Epidemiologia Psiquiátrica en México realizado en 2003, que formó parte de una iniciativa de la OMS, se describe la prevalencia de trastornos psiquiátricos, la comorbilidad, las variaciones en la distribución geográfica de los trastornos, los correlatos socio-demográficos y la utilización de servicios por la población adulta. Hay algunos datos relevantes que merecen ser mencionados para tener una idea del desequilibrio imperante entre oferta y demanda. Independientemente de que las cifras puedan haber variado un poco en los últimos años, las tendencias subsisten: el $28 \%$ de la población presentó alguno de los trastornos de la Clasificación Internacional de Enfermedades Mentales alguna vez en el transcurso de su vida; el 13\% lo reportó en los últimos 12 meses y el 5.8\% en los últimos 30 días. Por tipo de trastornos, los más frecuentes fueron los de ansiedad (14.3\%), alguna vez en el curso de su vida; seguidos por los trastornos de uso de sustancias (9.2\%) y los trastornos de la afectividad (9.1\%). ${ }^{7}$

Los hombres presentan prevalencias más altas de cualquier trastorno en comparación con las mujeres (30.4\% vs. $27.1 \%$ ), para alguna vez en la vida. Sin embargo, las mujeres presentan prevalencias globales más elevadas para cualquier trastorno en los últimos 12 meses (14.8\% vs. 12.9\%).

En México, uno de cada cinco individuos presenta al menos un trastorno mental en un momento de su vida. Los trastornos de ansiedad son los más prevalentes y los más crónicos; en tanto que los trastornos individuales más comunes son la depresión mayor, la fobia específica, la dependencia al alcohol y la fobia social.
Entre los hombres, la dependencia al alcohol es el problema más común, en tanto que entre las mujeres lo es la depresión mayor. La ansiedad por separación, el trastorno por déficit de atención y la fobia específica, son los padecimientos más tempranos y comunes en los niños.

Para los trastornos de la vida adulta, los de ansiedad se reportan con edades de inicio más tempranas, seguidos por los trastornos de la afectividad y por los de uso de sustancias.

Se estima que la Secretaría de Salud destina cerca del $2 \%$ de su presupuesto para la salud mental. El $80 \%$ de esta partida va a la operación de los hospitales psiquiátricos. Comparado con otros países latinoamericanos como Colombia, Brasil y Chile, el monto del presupuesto asignado para el rubro de salud mental es en ellos superior, pues oscila entre el 5\% y el 8\% del gasto total en salud. Es alentador constatar un incremento presupuestal de la SSa para este rubro en el último año, así como la estructuración de un nuevo programa de atención psiquiátrica a nivel nacional. ${ }^{8}$

Recientemente se aplicó el Instrumento de Evaluación para Sistemas de Salud Mental, de la Organización Mundial de la Salud (IESM-OMS), con el fin de recopilar información sobre el sistema de salud mental en México. Esto permitirá que nuestro país elabore planes de salud mental con objetivos claramente definidos, orientados a proporcionar mejores servicios comunitarios e involucrar más a los usuarios, a las familias y a otros sectores interesados en tareas de promoción, prevención, atención y rehabilitación. ${ }^{8}$

Destacan los planteamientos para integrar la salud mental a la red de servicios de salud en general, y la propuesta de revisar y actualizar la legislación en materia de salud mental, así como la protección de los derechos humanos de los pacientes y la protección social obligatoria para quienes sufren los trastornos mentales más frecuentes. ${ }^{9}$

Actualmente el eje de la atención de la salud mental es el tercer nivel de atención; es decir, el más especializado, que es también el más costoso. Hay 46 hospitales psiquiátricos, 13 unidades de internamiento psiquiátrico en hospitales generales y ocho establecimientos residenciales. La mayoría de estos servicios se ubican en las grandes ciudades o cerca a ellas. Actualmente existen 544 establecimientos de salud mental para pacientes ambulatorios que ofrecen atención a 310 usuarios por cada 100000 habitantes. El 27\% de la atención ambulatoria se proporciona a niños y adolescentes. ${ }^{6}$

Dada la elevada prevalencia de los trastornos mentales entre la población que requiere atención médica por diversas afecciones físicas que tienen repercusión en la esfera mental, es absolutamente necesario fortalecer el primer nivel de la atención médica. Se estima que cerca del 25\% de los pacientes que acuden a estos servicios pueden tener alguna patología psiquiátrica, que muchas veces no es reconocida y por lo tanto tampoco tratada. El motivo de esta falta de detección es el reflejo de múltiples factores, pero tal vez el más importante es la falta de una educación adecuada en el área de salud mental para los profesionales de la salud que, en general, están poco 
sensibilizados para detectar y atender a los pacientes que presentan alguna comorbilidad psiquiátrica.

En suma, con base en la información veraz disponible, quedan pocas dudas de que la demanda rebasa cotidianamente a la oferta, y que ésta, a pesar del esfuerzo realizado, es aún muy limitada en términos de infraestructura, recursos profesionales y financiamiento. ${ }^{10}$

\section{ESPECIALISTAS EN EJERCICIO}

De acuerdo con la Organización Mundial de la Salud (OMS), el equilibrio de recursos humanos en el sistema de salud es importante para alcanzar las metas de una buena atención. Una encuesta publicada en el 2005 mostró, en México, una mayor concentración de médicos generales y especialistas en las principales zonas urbanas, semejante a lo que ocurre en otros países. Es decir, que a mayor distancia con un centro urbano, menor será el número de especialistas

En el Atlas de Salud Mental de la OMS, publicado en ese mismo año, se observa una importante variación entre los diversos países según su nivel socio-económico; el número de psiquiatras, en cifras, expresadas por cada 100000 habitantes se observa en el cuadro $1 .^{11}$
Cuadro 1. Distribución de psiquiatras en diferentes países

\begin{tabular}{lcc}
\hline País & Habitantes & Psiquiatras/1000 000 \\
\hline Estados Unidos & 297043000 & 13.7 \\
Argentina & 38871000 & 13.2 \\
Canadá & 31743000 & 12.0 \\
Alemania & 82526000 & 11.8 \\
Brasil & 180655000 & 4.8 \\
España & 41128000 & 3.6 \\
México & 104931000 & 2.7
\end{tabular}

Promedio Mundial: 4.15 psiauiatras/100 000 Fuente: OMS, 2005.

En México, en un estudio nacional, se contabilizaron en 1988 un total de 1108 psiquiatras, lo cual, tomando en cuenta la población en ese año, sugiere que había 1.5 psiquiatras por cada 100000 habitantes. Ese estudio mostró también que el 56\% de los psiquiatras se encontraban en el Distrito Federal, con una tasa de 6.3 psiquiatras/100 000 habitantes en esta zona.

En un estudio más reciente, realizado en 2012, se contabilizaron un total de 3823 psiquiatras en nuestro país cuya población era de 112000000 habitantes; de éstos, 225 correspondían a la subespecialidad de paidopsiquiatría. Lo anterior da una tasa de 3.47 psiquiatras por cada 100000

Cuadro 2. Sedes de cursos formales de Residencia en Psiquiatría en la República Mexicana con reconocimiento universitario

\begin{tabular}{|c|c|c|}
\hline Entidad & Unidad hospitalaria & Universidad \\
\hline Baja California Norte & Instituto de Salud Mental de Baja California Norte & Universidad Autónoma de Baja California. \\
\hline Campeche & Hospital Psiquiátrico de Campeche & Universidad Autónoma de Campeche. \\
\hline \multirow[t]{6}{*}{ Distrito Federal } & $\begin{array}{l}\text { 1. Instituto Nacional de Psiquiatría Ramón de la Fuente } \\
\text { Muñiz }\end{array}$ & Universidad Nacional Autónoma de México. \\
\hline & $\begin{array}{l}\text { 2. Instituto Nacional de Neurología y Neurocirugía Ma- } \\
\text { nuel Velasco Suárez }\end{array}$ & \\
\hline & 3. Hospital Español de México & \\
\hline & 4. Centro Médico 20 de Noviembre ISSSTE & \\
\hline & $\begin{array}{l}\text { 5. Centro Médico Nacional Siglo XXI. Hospital de Espe- } \\
\text { cialidades. IMSS }\end{array}$ & \\
\hline & 6. Hospital Psiquiátrico Morelos & \\
\hline Durango & Hospital Psiquiátrico Miguel Vallebueno & Universidad Juárez del Estado de Durango. \\
\hline Guanajuato & Hospital Psiquiátrico San Pedro del Monte & Universidad de Guanajuato. \\
\hline \multirow[t]{3}{*}{ Jalisco } & 1. Hospital Civil de Guadalajara & Universidad de Guadalajara. \\
\hline & 2. Instituto Jalisciense de Salud Mental & Universidad Autónoma de Guadalajara. \\
\hline & 3. Hospital San Juan de Dios & \\
\hline \multirow[t]{3}{*}{ Nuevo León } & 1. Hospital Universitario de Nuevo León & Universidad Autónoma de Nuevo León. \\
\hline & 2. Hospital Psiquiátrico Nuevo León & Instituto Tecnológico y de Estudios Superiores de Monterrey \\
\hline & 3. Hospital Psiquiátrico del IMSS & Universidad de Monterrey. \\
\hline Puebla & Hospital Universitario de Puebla & Benemérita Universidad Autónoma de Puebla. \\
\hline San Luis Potosí & Clínica Everardo Neumann & Universidad Autónoma de San Luis Potosí. \\
\hline Sonora & Hospital Psiquiátrico Cruz del Norte & Universidad de Sonora. \\
\hline Tabasco & Hospital de Alta Especialidad de Salud Mental & Universidad Juárez Autónoma de Tabasco. \\
\hline Tamaulipas & Hospital Psiquiátrico de Tampico & Universidad Autónoma de Tamaulipas. \\
\hline Yucatán & Hospital Psiquiátrico de Yucatán & Universidad Autónoma de Yucatán. \\
\hline
\end{tabular}


Cuadro 3. XXXV Examen Nacional de Aspirantes a Residencias Médicas. 2011.

Puntajes máximos y mínimos

Plazas de médicos residentes mexicanos civiles (R-1)

\begin{tabular}{lcc}
\hline & \multicolumn{2}{c}{ Puntaje } \\
\cline { 2 - 3 } Especialidad & Máximo & Mínimo \\
\hline Anatomía patológica & 76.000 & 60.800 \\
Anestesiología & 79.000 & 61.550 \\
Audiología, otoneurología y foniatría & 75.550 & 61.775 \\
Calidad de la atención clínica & 75.550 & 56.875 \\
Cirugía general & 86.500 & 68.575 \\
Epidemiología & 66.600 & 58.875 \\
Genética médica & 78.300 & 64.950 \\
Geriatría & 78.800 & 65.575 \\
Ginecología y obstetricia & 81.375 & 65.500 \\
Imagenología diagnóstica y terapéutica & 79.525 & 61.775 \\
Medicina de rehabilitación & 78.050 & 64.950 \\
Medicina de urgencias & 78.575 & 63.725 \\
Medicina del trabajo y ambiental & 76.425 & 59.425 \\
Medicina familiar & 76.675 & 53.450 \\
Medicina integrada & 68.675 & 52.525 \\
Medicina interna & 83.825 & 67.775 \\
Medicina legal & 70.650 & 64.525 \\
Medicina nuclear & 69.700 & 62.050 \\
Neumología & 80.525 & 67.125 \\
Oftalmología & 82.200 & 68.625 \\
Otorrinolaringología y cirugía & 79.800 & 68.925 \\
de cabeza y cuello & 73.250 & 61.350 \\
Patología clínica & 81.750 & 63.775 \\
Pediatría & 78.200 & 65.225 \\
Psiquiatría & 74.350 & 63.750 \\
Radio oncología & 76.025 & 60.725 \\
Salud pública & 81.450 & 66.100 \\
\hline Traumatología y ortopedia & & \\
\hline
\end{tabular}

Fuente: Comisión Interinstitucional para la formación de recursos humanos para la salud, 2011.

habitantes y de 0.69 paidopsiquiatras por cada 100000 habitantes menores de 15 años. ${ }^{12}$
El $65 \%$ de los psiquiatras son hombres y $35 \%$ son mujeres. El 43\% ejerce su especialidad en el Distrito Federal con una tasa de 18.8 psiquiatras por 100000 habitantes; siguen después el Estado de Jalisco donde ejerce el 11.2\% de psiquiatras con una tasa de 5.8/100 000 habitantes y el de Nuevo León, donde se concentra el $6.46 \%$ con una tasa de 5.3 psiquiatras/100 000 habitantes. El 38.7\% restante se encuentra distribuido en las demás entidades federativas, principalmente en zonas urbanas (figura 1).

Es evidente que el número de médicos especializados en psiquiatría es insuficiente para cubrir las necesidades de la población en México, según la OMS. Entidades como el Distrito Federal, Jalisco y Nuevo León han cubierto o superado el promedio mundial, mientras que los Estados de Zacatecas, Chiapas y Tlaxcala cuentan con menos de 1 psiquiatra por cada 100000 habitantes. Por lo tanto, el problema no sólo es que hay pocos psiquiatras, sino que están muy mal distribuidos. Con los especialistas disponibles resulta imposible cubrir las necesidades de salud mental en nuestro país. ${ }^{12}$

\section{LAS RESIDENCIAS EN PSIQUIATRÍA}

Hace 61 años la Escuela de Graduados de la UNAM ofreció por primera vez un curso oficial de especialización en psiquiatría.

En la actualidad, el curso de especialización en Psiquiatría tiene una duración de cuatro años, se imparte en 22 instituciones y cuenta con el respaldo de 16 universidades (cuadro 2). Todos siguen los lineamientos del Plan Único de Especialidades Médicas (PUEM) de la UNAM. ${ }^{13}$

En 1971 inició el curso de subespecialidad en psiquiatría infantil y de la adolescencia, que dura dos años. Se desarrolla en tres sedes clínicas con reconocimiento de la UNAM y tiene como eje al Hospital Psiquiátrico Infantil Juan N. Navarro. ${ }^{3,14}$

Todos los aspirantes a cursar la especialidad de psiquiatría deben presentar el Examen Nacional de Aspirantes a Residencias Médicas y obtener un determinado puntaje para poder ocupar una de las plazas disponibles en el año por cursar. En el cuadro 3 se señalan los puntos máximos y mínimos que se obtuvieron para ingresar a la residencia, se-

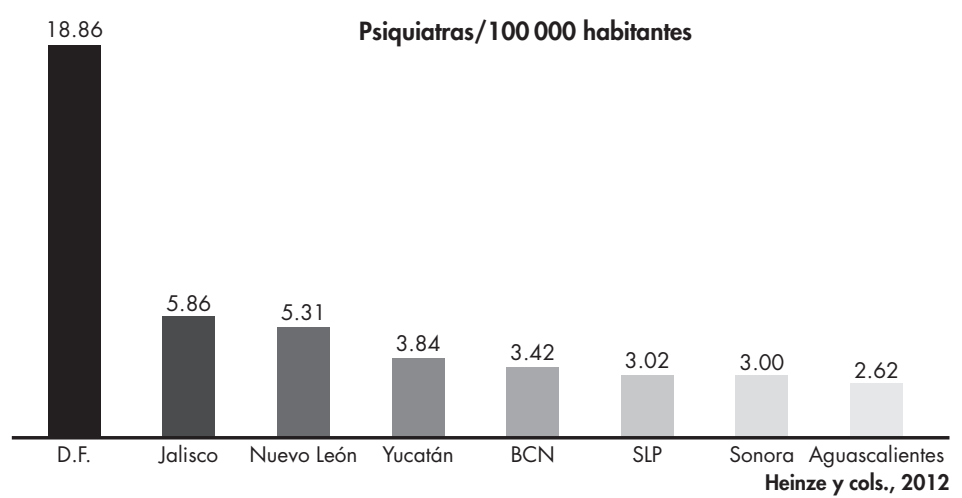

Figura 1. Distribución de psiquiatras en diversas entidades federativas. 
Cuadro 4. Programa curricular de la especialización en Psiquiatría

\begin{tabular}{|c|c|c|c|c|c|c|c|}
\hline \multicolumn{2}{|c|}{ Primer año } & \multicolumn{2}{|c|}{ Segundo año } & \multicolumn{2}{|c|}{ Tercer año } & \multicolumn{2}{|c|}{ Cuarto año } \\
\hline $\begin{array}{c}\text { Primer } \\
\text { semestre }\end{array}$ & $\begin{array}{l}\text { Segundo } \\
\text { semestre }\end{array}$ & $\begin{array}{c}\text { Tercer } \\
\text { semestre }\end{array}$ & $\begin{array}{c}\text { Cuarto } \\
\text { semestre }\end{array}$ & $\begin{array}{c}\text { Quinto } \\
\text { semestre }\end{array}$ & $\begin{array}{c}\text { Sexto } \\
\text { semestre }\end{array}$ & $\begin{array}{l}\text { Séptimo } \\
\text { semestre }\end{array}$ & $\begin{array}{c}\text { Octavo } \\
\text { semestre }\end{array}$ \\
\hline $\begin{array}{l}\text { Seminario } \\
\text { de atención } \\
\text { médica I }\end{array}$ & $\begin{array}{l}\text { Semanario } \\
\text { de atención } \\
\text { médica I }\end{array}$ & $\begin{array}{l}\text { Seminario } \\
\text { de atención } \\
\text { médica II }\end{array}$ & $\begin{array}{l}\text { Seminario } \\
\text { de atención } \\
\text { médica II }\end{array}$ & $\begin{array}{l}\text { Seminario } \\
\text { de atención } \\
\text { médica III }\end{array}$ & $\begin{array}{l}\text { Seminario } \\
\text { de atención } \\
\text { médica III }\end{array}$ & $\begin{array}{l}\text { Seminario } \\
\text { de atención } \\
\text { médica III }\end{array}$ & $\begin{array}{l}\text { Seminario } \\
\text { de atención } \\
\text { médica III }\end{array}$ \\
\hline $\begin{array}{l}\text { Psicopatología } \\
\text { médica I }\end{array}$ & $\begin{array}{l}\text { Psicopatolo- } \\
\text { gía II }\end{array}$ & $\begin{array}{l}\text { Psicopatolo- } \\
\text { gía III }\end{array}$ & $\begin{array}{l}\text { Psicopatología } \\
\text { IV }\end{array}$ & $\begin{array}{l}\text { Psicopatolo- } \\
\text { gía V }\end{array}$ & $\begin{array}{l}\text { Psicopatología } \\
\text { VI }\end{array}$ & $\begin{array}{l}\text { Reabilitación } \\
\text { del enfermo } \\
\text { psiquiátrico }\end{array}$ & $\begin{array}{l}\text { Psiquiatría } \\
\text { forense y crimi- } \\
\text { nología }\end{array}$ \\
\hline $\begin{array}{l}\text { Psicopatología } \\
\text { I: Introducción } \\
\text { a la psicopato- } \\
\text { logía }\end{array}$ & $\begin{array}{l}\text { Biología de } \\
\text { las funciones } \\
\text { mentales }\end{array}$ & $\begin{array}{l}\text { Abuso de } \\
\text { sustancias y } \\
\text { alcoholismo }\end{array}$ & $\begin{array}{l}\text { Psiquiatría } \\
\text { infantil y de la } \\
\text { adolescencia }\end{array}$ & Psicogeriatría & $\begin{array}{l}\text { Trastornos de } \\
\text { la sexualidad } \\
\text { y trastornos } \\
\text { psiquiátricos } \\
\text { relacionados } \\
\text { con el género }\end{array}$ & $\begin{array}{l}\text { Sistema de } \\
\text { salud mental }\end{array}$ & $\begin{array}{l}\text { Epidemiología } \\
\text { y psiquiatría } \\
\text { social y comu- } \\
\text { nitaria }\end{array}$ \\
\hline $\begin{array}{l}\text { Anatomía y } \\
\text { fisiología del } \\
\text { Sistema Ner- } \\
\text { vioso }\end{array}$ & $\begin{array}{l}\text { Neuropsico- } \\
\text { farmacología } \\
\text { clínica }\end{array}$ & $\begin{array}{l}\text { Neurología en } \\
\text { psiquiatría }\end{array}$ & $\begin{array}{l}\text { Psicoendo- } \\
\text { crinología e } \\
\text { inmunología }\end{array}$ & $\begin{array}{l}\text { Electroencefalo- } \\
\text { grafía }\end{array}$ & $\begin{array}{l}\text { Psicoterapia IV: } \\
\text { Casos clínicos }\end{array}$ & $\begin{array}{l}\text { Psicoterapia V:- } \\
\text { Casos clínicos }\end{array}$ & $\begin{array}{l}\text { Psicoterapia VI: } \\
\text { Casos clínicos }\end{array}$ \\
\hline $\begin{array}{l}\text { Urgencias en } \\
\text { psiquiatría }\end{array}$ & $\begin{array}{l}\text { Revisión biblio- } \\
\text { gráfica II }\end{array}$ & $\begin{array}{l}\text { Genética en } \\
\text { psiquiatría }\end{array}$ & $\begin{array}{l}\text { Psiquiatría } \\
\text { de hospital } \\
\text { general }\end{array}$ & $\begin{array}{l}\text { Imagenología } \\
\text { en psiquiatría }\end{array}$ & $\begin{array}{l}\text { Pruebas psico- } \\
\text { lógicas, neu- } \\
\text { ropsicológicas } \\
\text { y clinimetría }\end{array}$ & $\begin{array}{l}\text { Revisión biblio- } \\
\text { gráfica VII }\end{array}$ & $\begin{array}{l}\text { Revisión biblio- } \\
\text { gráfica VIII }\end{array}$ \\
\hline $\begin{array}{l}\text { Historia de la } \\
\text { psiquiatría }\end{array}$ & $\begin{array}{l}\text { Cesión de ca- } \\
\text { sos clínicos II }\end{array}$ & $\begin{array}{l}\text { Psicoterapia } \\
\text { I:Teoría }\end{array}$ & $\begin{array}{l}\text { Psicoterapia II: } \\
\text { Teoría }\end{array}$ & $\begin{array}{l}\text { Psicoterapia III: } \\
\text { Teoría clínica }\end{array}$ & $\begin{array}{l}\text { Psicoterapia IV: } \\
\text { Teoría clínica }\end{array}$ & $\begin{array}{l}\text { Sesión de ca- } \\
\text { sos clínicos VII }\end{array}$ & $\begin{array}{l}\text { Sesión de } \\
\text { casos clínicos } \\
\text { VIII }\end{array}$ \\
\hline $\begin{array}{l}\text { Taller de medi- } \\
\text { cina basada en } \\
\text { evidencias }\end{array}$ & $\begin{array}{l}\text { Taller de } \\
\text { profesionalismo } \\
\text { médico }\end{array}$ & $\begin{array}{l}\text { Revisión biblio- } \\
\text { gráfica III }\end{array}$ & $\begin{array}{l}\text { Revisión biblio- } \\
\text { gráfica IV }\end{array}$ & $\begin{array}{l}\text { Revisión biblio- } \\
\text { gráfica V }\end{array}$ & $\begin{array}{l}\text { Revisión biblio- } \\
\text { gráfica VI }\end{array}$ & $\begin{array}{l}\text { Trabajo de } \\
\text { atención médi- } \\
\text { ca VII }\end{array}$ & $\begin{array}{l}\text { Trabajo de } \\
\text { atención médi- } \\
\text { ca VIII }\end{array}$ \\
\hline $\begin{array}{l}\text { Revisión biblio- } \\
\text { gráfica I }\end{array}$ & $\begin{array}{l}\text { Trabajo de } \\
\text { atención médi- } \\
\text { ca II }\end{array}$ & $\begin{array}{l}\text { Cesión de ca- } \\
\text { sos clínicos III }\end{array}$ & $\begin{array}{l}\text { Cesión de ca- } \\
\text { sos clínicos IV }\end{array}$ & $\begin{array}{l}\text { Cesión de ca- } \\
\text { sos clínicos V }\end{array}$ & $\begin{array}{l}\text { Sesión de ca- } \\
\text { sos clínicos VI }\end{array}$ & & \\
\hline $\begin{array}{l}\text { Cesión de ca- } \\
\text { sos clínicos I }\end{array}$ & & $\begin{array}{l}\text { Trabajo de } \\
\text { atención médi- } \\
\text { ca III }\end{array}$ & $\begin{array}{l}\text { Trabajo de } \\
\text { atención médi- } \\
\text { ca IV }\end{array}$ & $\begin{array}{l}\text { Trabajo de } \\
\text { atención médi- } \\
\text { ca V }\end{array}$ & $\begin{array}{l}\text { Trabajo de } \\
\text { atención médi- } \\
\text { ca VI }\end{array}$ & & \\
\hline $\begin{array}{l}\text { Trabajo de } \\
\text { atención mé- } \\
\text { dica I }\end{array}$ & & & $\begin{array}{l}\text { Taller de } \\
\text { técnicas de } \\
\text { docencias }\end{array}$ & & & & \\
\hline
\end{tabular}

gún las diferentes especialidades y plazas disponibles para el año escolar $2011 .^{13,15,16}$
Las plazas ofertadas para médicos aspirantes a la especialidad en Psiquiatría fueron 141 en 2009, y subsecuen-

Cuadro 5. Programa curricular de la especialización en psiquiatría infantil y de la adolescencia

\begin{tabular}{|c|c|c|c|}
\hline \multicolumn{2}{|c|}{$\begin{array}{c}\text { Primer año } \\
\text { Seminario de atención médica I }\end{array}$} & \multicolumn{2}{|c|}{$\begin{array}{c}\text { Segundo año } \\
\text { Seminario de atención médica }\end{array}$} \\
\hline Primer semestre & Segundo semestre & Tercer semestre & Cuarto semestre \\
\hline $\begin{array}{l}\text { Conceptos generales, clasifica- } \\
\text { ción y nosología psiquiátrica in- } \\
\text { fantil }\end{array}$ & $\begin{array}{l}\text { Trastornos psiquiátricos en la in- } \\
\text { fancia y la adolescencia }\end{array}$ & Psicofarmacoterapia & $\begin{array}{l}\text { El niño y el adolescente en la co- } \\
\text { munidad }\end{array}$ \\
\hline $\begin{array}{l}\text { El desarrollo de la personalidad y } \\
\text { de las funciones psíquicas } \\
\text { El diagnóstico neurológico del } \\
\text { niño } \\
\text { Profesionalismo médico }\end{array}$ & $\begin{array}{l}\text { Clinimetría y pruebas psicológi- } \\
\text { cas }\end{array}$ & $\begin{array}{l}\text { Psicoterapia del niño y del ado- } \\
\text { lescente } \\
\text { La psiquiatría legal del niño y del } \\
\text { adolescente }\end{array}$ & \\
\hline
\end{tabular}

Fuente: Departamento de Desarrollo Curricular. Subdivisión de Especialidades Médicas. División de estudios de Posgrado. Facultad de Medicina. UNAM. 2008. 
temente disminuyeron a 137 en 2010 y a 130 en 2011. La UNAM ofrece también programas de maestría y doctorado en diferentes campos de la medicina clínica, la biomedicina y la sociomedicina, que incluyen a la psiquiatría, las neurociencias y la salud mental. ${ }^{16}$

En los cuadros 4 y 5 se muestran las unidades didácticas para los cursos de especialización tanto de psiquiatría como de psiquiatría infantil y de la adolescencia de la UNAM. Ambos se encuentran en revisión permanente, así como la idoneidad de las sedes clínicas, por parte de los comités académicos correspondientes. ${ }^{16}$

\section{PERSPECTIVAS DE LA PSIQUIATRÍA COMO ESPECIALIDAD MÉDICA}

Los avances recientes en la psiquiatría son consecuencia del desarrollo de las neurociencias y del afianzamiento de sus ligas con el resto de la medicina. El progreso ha sido posible también por el desarrollo de técnicas, métodos e instrumentos innovadores que han enriquecido el campo de la psiquiatría clínica y que han permitido apreciar, en su justa dimensión epidemiológica y social, el impacto de los trastornos mentales.

La posibilidad de relacionar cada vez con más precisión los trastornos mentales con sus bases biológicas ha aumentado en forma notable. La imagenología, por ejemplo, ha permitido el estudio cada vez más fino de las estructuras del Sistema Nervioso Central y de su funcionamiento; en tanto que la genómica y la proteómica están abriendo nuevos horizontes en relación a la etiología y el tratamiento de algunas de las principales enfermedades mentales.

Los cambios que ha experimentado la psiquiatría en las últimas décadas son profundos y se expresan en los niveles conceptual, clínico y asistencial; abarcan desde la genética hasta la psicodinamia, pasan por la infancia, el ámbito familiar y social, e inciden en la terapéutica. ${ }^{17}$

Los períodos de hospitalización de los enfermos mentales se han reducido espectacularmente, y las posibilidades de reintegración a una vida social y productiva son mejores que nunca antes en la historia. No obstante, todavía hay mucho camino por recorrer y seguramente los avances científicos y el desarrollo de nuevos instrumentos técnicos permitirán, en los próximos años, incrementar la eficacia de las intervenciones de prevención primaria y secundaria.

La gran expectativa sigue siendo la posibilidad de actuar sobre la mente a través del cerebro, mediante substancias que tengan acciones específicas sobre el pensamiento, la memoria, el afecto, la sexualidad, el apetito, etcétera, y de influir en forma selectiva y radicalmente nueva sobre la conducta, los estados de conciencia, el humor y la memoria. Esta posibilidad aumenta conforme avanzan los conocimientos sobre las bases moleculares de las funciones mentales.

No hay duda de que la psiquiatría se ha convertido en una disciplina cada vez menos especulativa y más observante y experimental. Esto no significa que su campo deba reducirse al conocimiento del cerebro. Es necesario mantener vigentes las dimensiones psicológica y social para la comprensión cabal y el manejo eficaz de los enfermos. Pero hay que evaluar con rigor el peso patogénico real de los diversos factores que condicionan los cuadros clínicos propios de la psiquiatría y que con frecuencia determinan el éxito de las intervenciones psiquiátricas.

Conviene recordar, para concluir, las palabras del maestro Ramón de la Fuente: "A mi juicio, el valor de la psiquiatría actual no sólo radica en que es más científica y experimental sino también en su perspectiva, en su marco de orientación más amplio y coherente que no pierde de vista el lado subjetivo y social de los predicamentos humanos". Tal aseveración mantiene plena vigencia. ${ }^{5}$

\section{REFERENCIAS}

1. Calderón-Narváez G. Las enfermedades mentales en México. México: Editorial Trillas; 2002.

2. Ramírez-Moreno S. La asistencia psiquiátrica en México. En: Congreso Internacional de Psiquiatría. París: Secretaría de Salubridad y Asistencia; 1950.

3. De la Fuente R. Las especialidades médicas en México: Psiquiatría. En: Soberón G, Kumat J, Laguna J (Comps.). México: Biblioteca de la Salud, Fondo de Cultura Económica; 1989.

4. Pérez-Rincón H. Breve historia de la psiquiatría en México. México: Instituto Nacional de Psiquiatría; 1995.

5. De la Fuente R, De la Fuente JR. Psiquiatría en medicina. En: Tratado de medicina interna. Vol. 2. México: Academia Nacional de Medicina. El Manual Moderno; 1993.

6. De la Fuente R, Díaz A, Fouilloux C. La formación de psiquiatras en la República Mexicana. Salud Mental 1998;11:3-8.

7. Medina-Mora ME. Prevalencia de trastornos mentales y uso de servicios: Resultados de la Encuesta Nacional de Epidemiología Psiquiátrica en México. Salud Mental 2003;26:1-16.

8. Informe sobre el sistema de salud mental en México. México: IESMOMS; 2011.

9. Katsching $\mathbf{H}$. Are psychiatrists an endangered species? Observations on internal and external challenges to the profession. World Psychiatry 2010;9:21-28.

10. Okie S. The evolving care psysician. N Engl J Med 2012;366:1849-1853.

11. World Health Organization. Ginebra: Mental Health Atlas; 2005.

12. Heinze G, Chapa GC, Santisteban JA, Vargas I. Los médicos psiquiatras en México: Su distribución, ejercicio profesional y certificación. Salud Mental 2012;35:279-285.

13. Comisión Interinstitucional para la formación de recursos humanos para la salud. México: Dirección General de Calidad y Educación en Salud, SSA; ENARM 2009, 2010, 2011.

14. Plan único de especializaciones médicas en psiquiatría. Subdivisión de Especialidades Médicas. México: División de Estudios de Posgrado, Facultad de Medicina, UNAM; 2012.

15. Graue Wiechers E et al. Educación en las residencias médicas. México: Editores de Textos Mexicanos; 2010.

16. Plan Único de Especializaciones Médicas en Psiquiatría Infantil y de la Adolescencia. Subdivisión de Especialidades Médicas. México: División de Estudios de Posgrado. Facultad de Medicina, UNAM; 2008.

17. Eisenberg L. The social construction of the human brain. Am J Psychiatry 1990;152:1563-1575.

Artículo sin conflicto de intereses 